\title{
Contribución metodológica de Juan Antonio Tonda Magallón al cálculo estructural de las cáscaras cilíndricas largas de cubierta
}

\section{Methodological contribution of Juan Antonio Tonda Magallón to structural calculation of roof long cylindrical shells}

\author{
M. Martínez ${ }^{(*)}$ \\ RESUMEN
}

El arquitecto español Juan Antonio Tonda Magallón, es conocido como uno de los más importantes discípulos y colaboradores del arquitecto Félix Candela Outeriño.

A pesar de los numerosos escritos existentes sobre la vida y obra de Félix Candela, faltan estudios de detalle referentes a los métodos de cálculo empleados en sus estructuras. Tonda participó, sobretodo, en el cálculo estructural y desarrollo geométrico de las estructuras proyectadas por Candela; especializándose en las cáscaras delgadas de hormigón armado. En este artículo se analiza y desarrolla la aportación metodológica de J. A. Tonda al cálculo estructural de estas tipologías, concretamente al caso de las cáscaras cilíndricas largas de cubierta, basado en el análisis límite con el enfoque plástico del equilibrio. Con el método, a continuación expuesto y denominado método de la viga, se logró poder calcular, de manera sencilla y segura, estas tipologías estructurales como se tratará de demostrar a continuación.

Palabras clave: Juan Antonio Tonda Magallón; método de la viga; cálculo estructural de cáscaras cilíndricas largas; Félix Candela Outeriño.

\section{ABSTRACT}

The Spanish architect Juan Antonio Magallón is known as one of the most important disciples and collaborators of the architect Felix Candela Outeriño.

Despite many existing writings about Felix Candela's life and work, there is a lack of detailed studies concerning the calculation methods used in their structures. Tonda participated especially in structural design and geometric development of structures designed by Candela; specializing in thin reinforced concrete shells.

This article analyzes and develops methodological contribution of J.A. Tonda structural calculation of these types, specifically the case of roof long cylindrical shells, based on the limit analysis with plastic approach from equilibrium.

With the method, then exposed and called beam method, it was possible to calculate, safely and easily, these structural types as it is show below.

Keywords: Juan Antonio Tonda Magallón; beam method; structural calculation of roof long cylindrical shells; Félix Candela Outeriño.

(*) Escuela de Arquitectura. Universidad de Alcalá de Henares

Persona de contacto/Corresponding author: martinezmartinezmonica@gmail.com (M. Martínez)

ORCID: http://orcid.org/oooo-0oo3-4544-9517 (M. Martïnez)

Cómo citar este artículo/Citation: M. Martínez. (2018). Contribución metodológica de Juan Antonio Tonda Magallón al cálculo estructural de las cáscaras cilíndricas largas de cubierta. Informes de la Construcción, 70(550): e249. https://doi.org/10.3989/ic.16.078 Copyright: (c) 2018 CSIC. Este es un artículo de acceso abierto distribuido bajo los términos de la licencia de uso y distribución Creative Commons Reconocimiento 4.0 Internacional (CC BY 4.0). 


\section{INTRODUCCIÓN}

Las cáscaras cilíndricas largas de cubierta, de hormigón armado, comienzan a tipificarse una vez terminada la Segunda Guerra Mundial.

En sus orígenes, evaluar el estado tensional de estas tipologías pasaba por aplicar la teoría de la elasticidad, firmemente establecida en este periodo. La aplicación del método elástico al cálculo estructural de las cáscaras cilíndricas largas fue desarrollada en el decenio de 1930, en Alemania, principalmente por los ingenieros U. Finsterwalder (1) y Fr. Dischinger (2), y posteriormente por el noruego A. Aas Jakobsen (3).

La aplicación del análisis elástico consistía en precisar una serie de hipótesis, concernientes a las condiciones de contorno de la estructura a analizar o/y del material estructural utilizado. Todas estas hipótesis suponían, o bien idealizar una realidad imposible de conocer a priori, o bien hacer referencia a un material ideal, homogéneo e isótopo, cuando el hormigón armado no cumplía con ninguna de estas propiedades. Sin embargo, no se podía asegurar que ese estado tensional, obtenido en la cáscara, representara el "estado real" indiscutible de la estructura. Por todo ello, el resultado del análisis elástico en una estructura no podía ser observado en la realidad; siendo lo verdaderamente importante a tratar, puesto que era lo que había que evitar, estudiar las condiciones en las que se producía el colapso de ésta.

Un marco teórico más adecuado al proporcionado por la teoría elástica, para el cálculo estructural de las cáscaras cilíndricas largas, resultó ser el análisis a rotura. Aunque el estado "real" de la estructura no se podía conocer, la resistencia de ésta sí se podía calcular con precisión; siendo, además, muy insensible a las supuestas imperfecciones de fabricación y ejecución o a pequeñas variaciones en las condiciones de contorno.

En este contexto, aparece la figura del ingeniero danés Knud Winstrup Johansen; el cual en 1944 publicó un artículo de gran relevancia, en cuanto que marcó el origen de la aplicación del análisis límite al cálculo estructural de las cáscaras cilíndricas largas de cubierta, con un enfoque plástico del equilibrio (4). Posteriormente, otros expertos en plasticidad como el también ingeniero danés H. Lundgren (5) y el húngaro G.v. Kazinczy (6) continuaron con este tipo de estudios.

A partir de 1950, y hasta la aparición de los ordenadores, con la teoría de la plasticidad firmemente establecida y sus teoremas demostrados, el comportamiento estructural de las cáscaras cilíndricas largas de cubierta se analiza en base a la obtención de diferentes estados de equilibrio, haciendo hincapié en la seguridad, respetando la característica de cedencia del hormigón armado y, por tanto, obviando las consideraciones de compatibilidad y deformación que pudiera sufrir la estructura, tal y como determinaba la teoría de la elasticidad.

De esta manera es como actuaron algunos calculistas del momento, como fue el caso de Juan Antonio Tonda Magallón.

El arquitecto español J.A. Tonda, nacido en Madrid en 1931 y actualmente profesor de la Universidad Nacional Autónoma de México (UNAM), es conocido como uno de los más importantes discípulos y colaboradores del arquitecto Félix Candela. Pertenece a la llamada "generación hispano-mexicana", formada por niños y jóvenes que llegaron a México acompa- ñando el éxodo de sus padres y formándose profesionalmente en su patria de adopción.

Tonda se caracterizó por ser el discípulo y colaborador más influyente de la obra de Candela. Su labor, fundamentalmente, se basó en el estudio del desarrollo geométrico y en el cálculo de sus estructuras. Por ello, Tonda logró especializarse en el cálculo estructural de cáscaras delgadas de hormigón armado; aplicando en el caso de las cáscaras cilíndricas largas un método de cálculo, denominado método de la viga, basado en su conocimiento adquirido sobre el análisis límite con el enfoque plástico del equilibrio.

\section{DESARROLLO DEL MÉTODO DE LA VIGA POR JUAN ANTONIO TONDA, APLICADO AL CÁLCULO ESTRUCTURAL DE LAS CÁSCARAS CILÍNDRICAS LARGAS}

Siendo profesor del Instituto Mexicano del Cemento y del Hormigón (IMCYC), Juan Antonio Tonda Magallón escribe el libro Cascarones de Concreto (7), publicado en 1974, gracias a la colaboración mutua entre el IMCYC y el Instituto de Investigaciones Arquitectónicas de la UNAM. En este documento, Tonda expone el método utilizado para el análisis y cálculo estructural de las cáscaras cilíndricas largas de cubierta proyectadas, por tanto, por Félix candela.

Tonda señala que para realizar el cálculo de estas tipologías estructurales debe emplearse el método de la viga. Este método, desarrollado por el ingeniero danés H. Lundgren en su libro publicado en 1949 (5), fue enunciado y publicado por primera vez por el también ingeniero danés K. W. Johansen, en 1944 (4). Johansen fundamentó el método de la viga, aplicado al cálculo estructural de las cáscaras cilíndricas largas de cubierta, desde el punto de vista del análisis límite con un enfoque plástico del equilibrio de fuerzas.

Tonda define el método de la viga como un método basado en la hipótesis de que la cáscara cilíndrica larga funciona como si de una viga recta, hueca y de sección transversal circular se tratara. Por tanto, la cáscara se calcula longitudinal y transversalmente, en su conjunto, al igual que si de una viga se tratara.

Tonda califica al método de la viga como el método que aúna tanto seguridad como sencillez en el desarrollo matemático del cálculo estructural de la cáscara cilíndrica larga; en contra de la complejidad intrínseca que entrañaba la teoría elástica o de la teoría de la membrana; la cual, esta última, no tenía en cuenta el cálculo de los momentos transversales que sí se originan en la cáscara y que, por lo tanto, requerían armadura de refuerzo.

\subsection{Desarrollo del cálculo}

Partiendo de una cáscara cilíndrica larga, de longitud $L$ y de directriz circular (Figura 1), Juan Antonio Tonda procede, en primer lugar, al cálculo longitudinal de la cáscara para continuar con el cálculo transversal de ésta.

\section{Cálculo longitudinal de la cáscara}

El cálculo comienza hallando el peso de una franja de la cáscara cilíndrica, de un metro de ancho en sentido longitudinal, cuyo valor vendrá dado por la expresión conocida:

$$
\omega_{b}=2 \omega \alpha R
$$




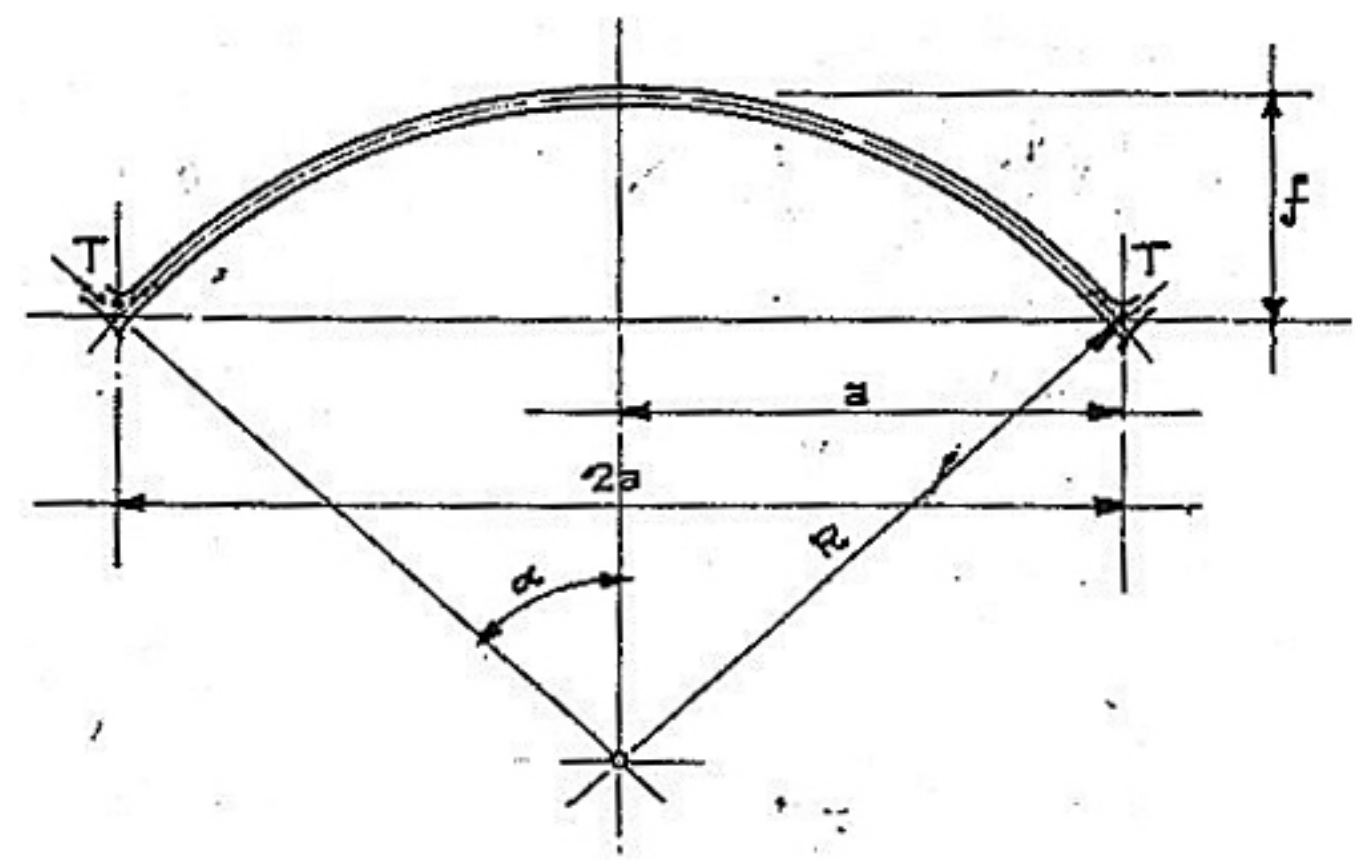

Figura 1. Sección transversal de la cáscara cilíndrica larga a calcular. Tonda Magallón J.A. (1974). Cascarones de Concreto. Capítulo V, figura 77, p. 42.

Al tomar el ángulo $\alpha$ en radianes, y asimilando la geometría de la cáscara cilíndrica al de una viga, el momento flector total vendría dado por la siguiente expresión:

$$
M=\frac{\omega_{b} L^{2}}{8}
$$

El valor de este momento flector es resistido por las fuerzas internas; por lo que habrá que calcular la magnitud de éstas para, posteriormente, hallar la cantidad de refuerzo necesario en la cáscara.

Con el fin de hallar las fuerzas internas, Tonda procede a determinar el brazo de palanca de la cáscara por medio de expresiones sumamente sencillas, derivadas de la propia geometría de la cáscara a estudiar. Para ello, procede a calcular el centro de las compresiones y de las tracciones ubicadas en la sección transversal de la cáscara, de la siguiente manera:

El momento $\mathrm{M}$ debe ser resistido por la acción de fuerzas internas en la cáscara; de compresión y de tracción. En relación a las compresiones, Tonda las supone contenidas en una longitud de cáscara, aproximadamente de 1/3 a 1/4 de la longitud transversal de la cáscara (Figura 2) y de expresión:

$$
S_{c}=\left(\frac{1}{3} \dot{o} \frac{1}{4}\right) S_{b}
$$

siendo:

$$
S_{b}=2 \alpha R
$$

Este área constituirá la zona de compresión, por lo que su centro de gravedad corresponderá al centro de aplicación de las fuerzas de compresión.

El centro de gravedad de la zona de compresiones viene dado por la expresión:

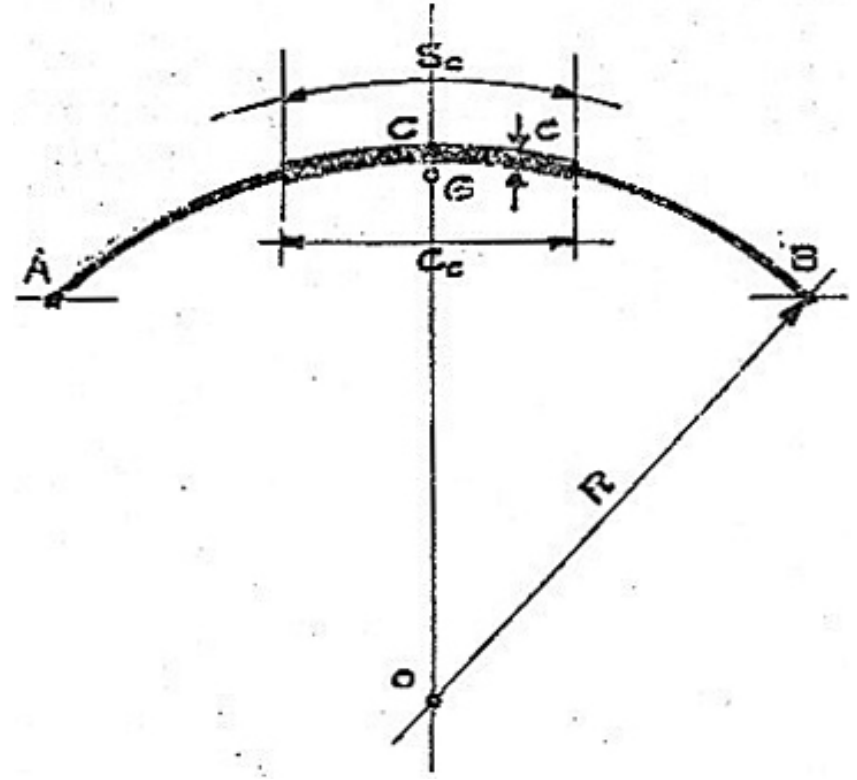

Figura 2. Sección transversal de la cáscara cilíndrica larga. Tonda Magallón J.A. (1974). Cascarones de Concreto. Capítulo V, figura 78, p. 43.

$$
\overline{O G}=R \frac{C_{c}}{S_{c}}
$$

donde el valor de la distancia CG, responde a la expresión:

$$
\overline{C G}=R-\overline{O G}=R\left(1-\frac{C_{c}}{S_{c}}\right)
$$

mientras que la magnitud de la distancia $\mathrm{Cc}$ es determinada por sencillas razones trigonométricas del caso concreto de cáscara a calcular.

El punto G representa el centro de las compresiones; mientras que el centro de las tracciones, Tonda, las supone a $5 \mathrm{~cm}$ 
por encima de los puntos A y B (Figura 2). En base a ello, el brazo de palanca de las fuerzas de compresión y tracción vendrá determinado por la expresión siguiente (Figura 3):

$$
Z=f-\overline{C G}-0,05 m=f-R\left(1-\frac{C_{c}}{S_{c}}\right)-0,05 m
$$

En el caso de aparecer momentos negativos, existentes en los puntos de unión entre cáscaras cilíndricas largas contiguas, Tonda procede al cálculo del brazo de palanca, Z, de idéntica manera; es decir, a través de expresiones sencillas obtenidas a partir de la propia geometría de la cáscara a calcular.

Una vez obtenido el brazo de palanca, $Z$, el valor las fuerzas internas de compresión se determina, fácilmente, mediante la expresión:

$$
F=\frac{M}{Z}
$$

siendo $\mathrm{F}$ el valor de las fuerzas, tanto de compresión como de tracción.

Una vez obtenido el valor de estas fuerzas, y antes de proseguir con el desarrollo matemático, Tonda señala que debe comprobarse que la tensión unitaria del hormigón cumple con la siguiente condición:

$$
\sigma_{b} \leq 0,30 f_{c}^{\prime}
$$

donde:

$$
\sigma_{b}=\frac{F}{S_{c} e}
$$

Una vez constatada dicha condición, el área necesaria de refuerzo queda determinada mediante la expresión:

$$
F_{e}=\frac{F}{\sigma_{e}}
$$

Este refuerzo se distribuirá por igual en las dos zonas inferiores de la cáscara, en los centros de tracción (Figura 4).

Por tanto, el cálculo longitudinal de la cáscara cilíndrica así realizado se ha desarrollado bajo la hipótesis de estado plástico puesto que los esfuerzos de compresión se han considerado repartidos uniformemente en la zona supuesta (Figura 4) y basándose en un análisis de equilibrio de fuerzas.

Cálculo transversal de la cáscara

\section{Cálculo de las fuerzas transversales:}

A la distribución de esfuerzos de compresión y tracción (Figura 5a), actuando en la cáscara, también hay que añadir

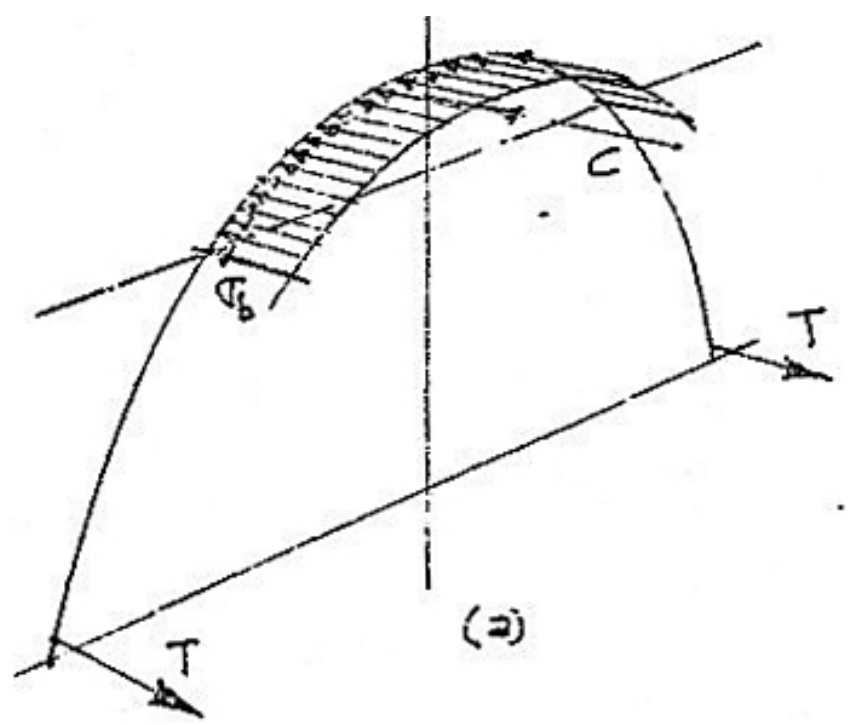

Figura 4. Área de compresiones en la sección transversal de la cáscara cilíndrica larga. Tonda Magallón J.A. (1974). Cascarones de Concreto. Capítulo V, figura 80a, p. 44.

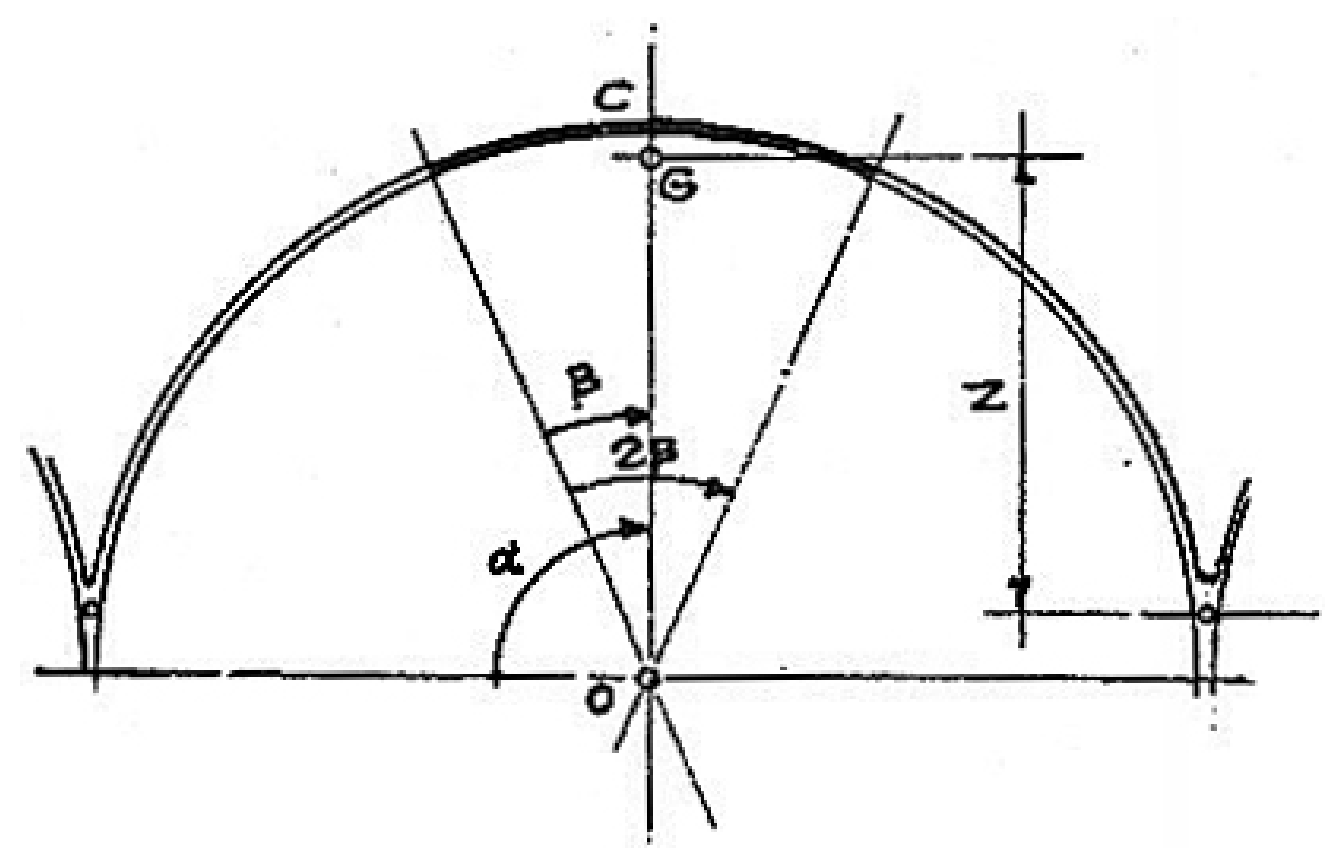

Figura 3. Brazo de palanca en la sección transversal de la cáscara cilíndrica larga. Tonda Magallón J.A.

(1974). Cascarones de Concreto. Capítulo V, figura 93, p. 53. 

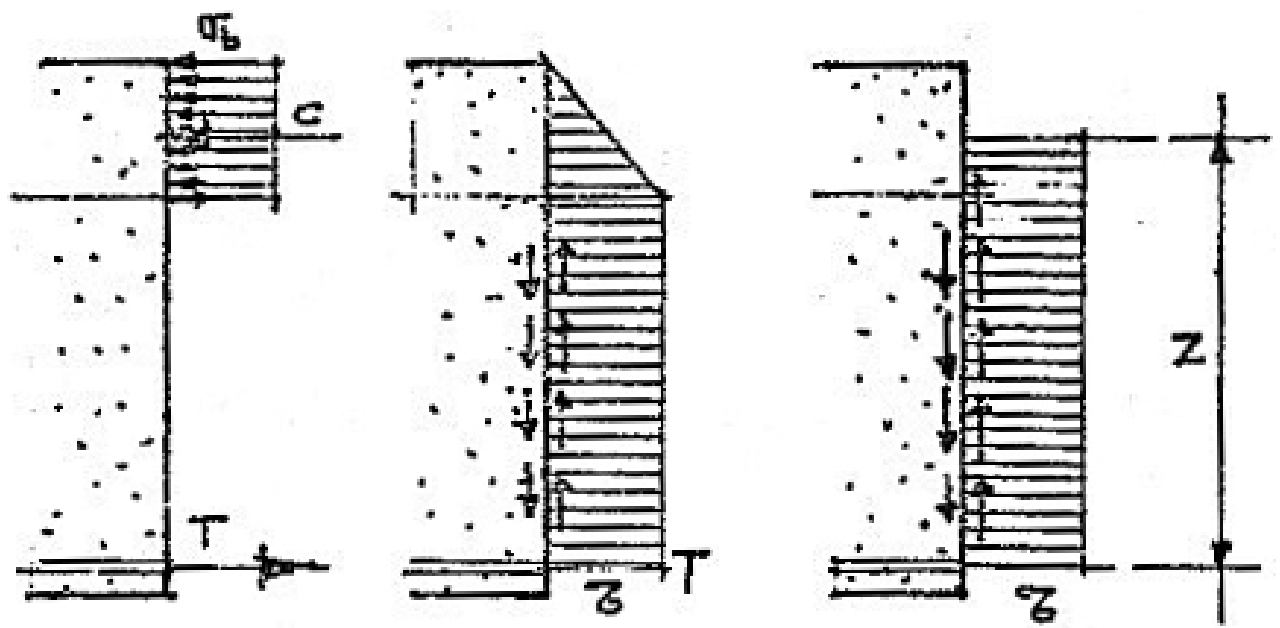

Figura 5. Distribución de esfuerzos en la sección transversal de la cáscara cilíndrica larga. Tonda Magallón J.A. (1974). Cascarones de Concreto. Capítulo V, figura 8ob, c y d, p. 44.

los esfuerzos tangenciales y los cortantes (Figura 5b). A su vez, los esfuerzos tangenciales pueden ser sustituidos por una distribución homogénea, con el fin de simplificar los cálculos, a lo largo de todo el brazo de palanca calculado anteriormente, tal y como aparece esquematizado en la figura $5 \mathrm{c}$.

La suma de los esfuerzos tangenciales unitarios será la resultante que actúa en la sección, es decir:

$$
t=\frac{V_{n}}{2 Z}=\frac{\omega_{b}}{2 Z}
$$

siendo Vn la fuerza cortante que equilibra el peso propio de la cáscara y la reacción que compensa cada franja de ésta.

En base a lo expuesto, en primer lugar se analizarán los diferentes momentos transversales, que se originan en la sección transversal de la cáscara, para posteriormente calcular los esfuerzos cortantes.

\section{Cálculo de los momentos transversales:}

Tonda considera estudiar los siguientes momentos transversales, originados por sus correspondientes esfuerzos:

- Momentos transversales debidos al peso propio de la estructura.

- Momentos transversales debidos a los esfuerzos tangenciales.

- Momentos transversales debidos a la fuerza horizontal o/y fuerza vertical en el arranque de la cáscara.

A continuación se procederá a desarrollar cada uno de ellos, según la metodología planteada por J.A. Tonda (7).

2.1. Momentos transversales debidos al peso propio de la cáscara.

Estos momentos transversales se calculan por medio de la estática, considerando el equilibrio de una franja transversal de la cáscara cilíndrica larga, de longitud unitaria. Esta franja de estructura se encontrará sometida a las cargas verticales que actúan sobre ella y a la diferencia entre los esfuerzos cortantes en ambas secciones transversales que la delimitan.

Para ello Tonda considera una banda de cáscara de 1m de ancho (Figura 6), para realizar su estudio.
Los momentos transversales debidos al peso propio de la estructura vendrán dados por la siguiente sencilla expresión:

$$
M_{\varphi}=W_{\varphi} d
$$

donde:

$\mathrm{M} \varphi$ es el momento transversal debido al peso propio de la cáscara, $\mathrm{W} \varphi$, en un punto genérico de ésta. Dicho punto, $\mathrm{x}$, formará un ángulo $\varphi$ con la recta OA a partir del punto de arranque A de la cáscara (Figura 5).

$\mathrm{W} \varphi$, es el peso propio de la cáscara perteneciente a la longitud de $\operatorname{arco} S \varphi$ (Figura 6) y viene determinado por la expresión:

$$
W_{m}=S_{m} \omega=\varphi \omega R
$$

siendo:

$$
S_{\varphi}=\varphi R
$$

donde $d$ es la distancia de la resultante del peso propio de la cáscara, $\mathrm{W} \varphi$, al punto genérico de ésta, $x$ (Figura 6).

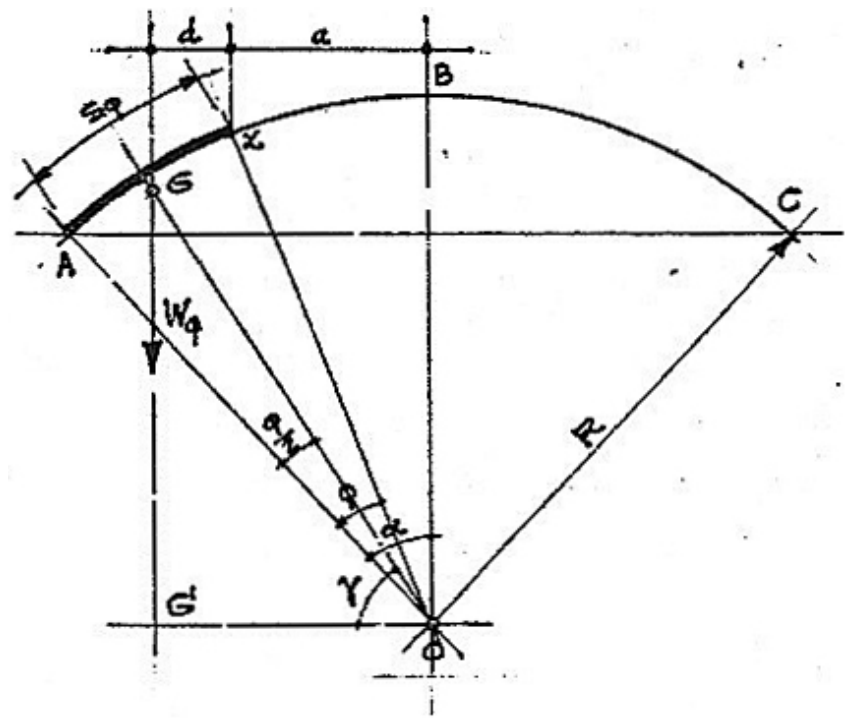

Figura 6. Determinación de momentos transversales debidos al peso propio de la cáscara cilíndrica larga. Tonda Magallón J.A. (1974). Cascarones de Concreto. Capítulo V, figura 84, p. 46. 
Dada la sencillez de la expresión, lo único que hay que averiguar es el punto de gravedad del área de la cáscara, $\mathrm{S} \varphi$, por donde pasará la resultante del peso propio y el valor de la distancia $d$.

Tonda obtiene ambos datos, de nuevo, a partir de la propia geometría de la cáscara, por medio de expresiones sumamente sencillas, como a continuación se exponen:

La distancia OG vendría dada por la expresión:

$$
\overline{O G}=R \frac{C_{\varphi}}{S_{\varphi}}
$$

donde $\mathrm{C} \varphi$ representa la cuerda del arco $\mathrm{S} \varphi$, y viene definida por la fórmula (Figura 6):

$$
C_{\varphi}=2 r \operatorname{sen} \varphi / 2
$$

que sustituyendo en la expresión [16], se obtiene:

$$
\overline{O G}=\frac{R 2 \operatorname{sen} \varphi / 2}{\varphi}
$$

Para el cálculo de la distancia $d$, Tonda considera el ángulo $\gamma$ que forma la recta OG con la horizontal OG' (Figura 6) y que tiene un valor de:

$$
\gamma=90^{\circ}-\alpha+\frac{\varphi}{2}=90^{\circ}-\left(\alpha-\frac{\varphi}{2}\right)
$$

La distancia $d$ vendrá dada, por lo tanto, por la expresión:

$$
d=\overline{O G^{\prime}}-a
$$

donde (Figura 6):

$$
\operatorname{sen}(\alpha-\varphi)=\frac{a}{R} ; \quad a=R \operatorname{sen}(\alpha-\varphi)
$$

Proyectando horizontalmente la distancia OG, para obtener OG', se obtiene:

$$
\overline{O G^{\prime}}=\overline{O G} \cos \gamma=\overline{O G} \cos \left(90-\left(\alpha-\frac{\varphi}{2}\right)\right)=\overline{O G} \operatorname{sen}\left(\alpha-\frac{\varphi}{2}\right)[22]
$$

Y sustituyendo en la expresión [18], se consigue:

$$
\overline{O G^{\prime}}=R \frac{2 \operatorname{sen}^{\varphi} / 2}{\varphi} \operatorname{sen}(\alpha-\varphi / 2)
$$

Finalmente, sustituyendo las expresiones [21] y [23] en la fórmula [20], se obtiene el valor de la distancia $d$ :

$$
d=R\left[\frac{2 \operatorname{sen}^{\varphi} / 2}{\varphi} \operatorname{sen}(\alpha-\varphi / 2)-\operatorname{sen}(\alpha-\varphi)\right]
$$

Hallada la expresión del valor de la distancia, $d$, y sustituyendo en la expresión [13], el momento transversal debido al peso propio de la cáscara será:

$$
M_{\varphi}=\varphi \omega R^{2}\left[\frac{2 \operatorname{sen}^{\varphi} / 2}{\varphi} \operatorname{sen}(\alpha-\varphi / 2)-\operatorname{sen}(\alpha-\varphi)\right]
$$

y simplificando, la expresión definitiva sería:

$$
M_{\varphi}=-\omega R^{2}[\cos (\alpha-\varphi)-\cos \alpha-\varphi \operatorname{sen}(\alpha-\varphi)]
$$

2.2. Momentos transversales debidos a los esfuerzos tangenciales.

Para obtener los momentos debidos a los esfuerzos transversales primero se debe calcular la posición de la resultante de estas fuerzas, para una longitud de arco $\mathrm{S} \varphi$ y una sección cualquiera de la cáscara, $x$ (Figura 7).

Dicha resultante vendrá dada por la expresión:

$$
R_{t}=t C_{\varphi}=2 R \operatorname{tsen} \frac{\varphi}{2}
$$

Una vez obtenida la resultante de los esfuerzos tangenciales, Rt, el siguiente paso para obtener el momento transversal es determinar la distancia, $d$, a la cual se encuentra de la sección $x$ (Figura 8). Para ello, Tonda realiza el siguiente procedimiento:

Tomando momentos de las fuerzas tangenciales unitarias, $t$, respecto al centro de la circunferencia, punto $\mathrm{O}$ que conforma la sección transversal de la cáscara, se puede calcular la distancia, D, a la que se encuentra la resultante Rt del punto O (Figura 8).

Esta distancia vendrá dada, por tanto, por la expresión:

$$
D=\frac{M_{o}}{R_{t}}
$$

Tomando momento:

$$
M_{o}=\int_{0}^{\varphi} R t d s=\int_{0}^{\varphi} R t R d \varphi=\int_{0}^{\varphi} R^{2} t d \varphi=R^{2} t \varphi
$$

Sustituyendo las expresiones [27] y [29] en [28], se obtiene:

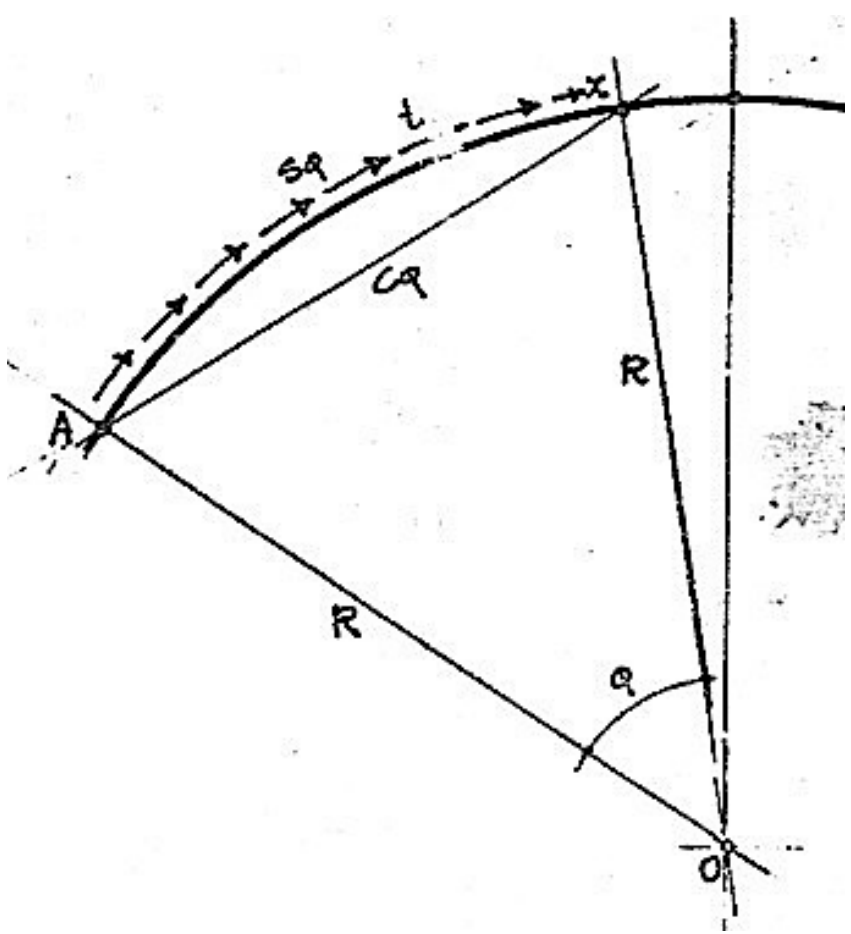

Figura 7. Distribución de las fuerzas tangenciales en la cáscara cilíndrica larga. Tonda Magallón J.A. (1974). Cascarones de Concreto. Capítulo V, figura 85, p. 48. 


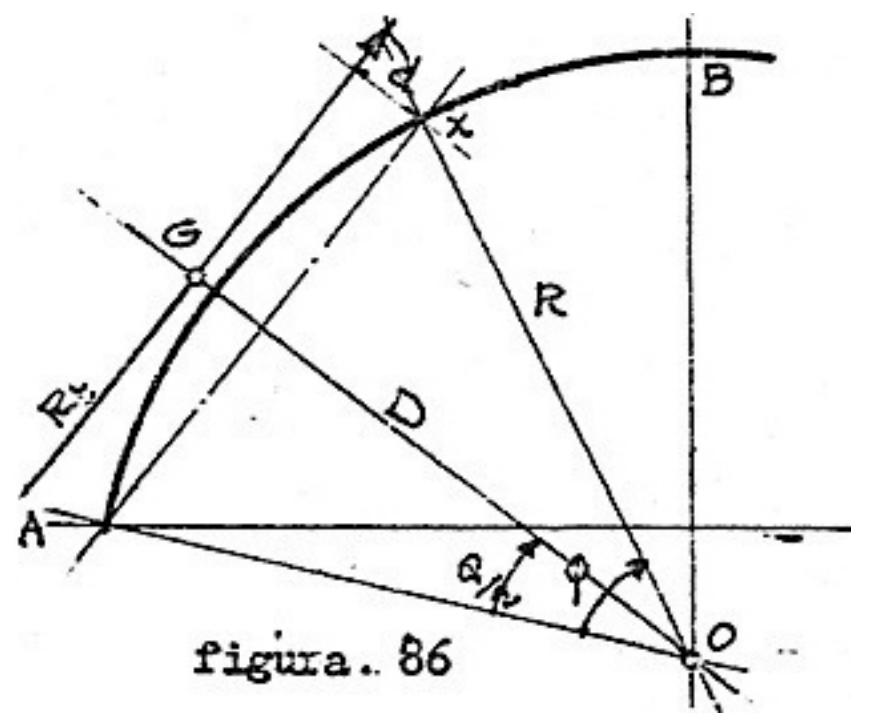

Figura 8. Resultante de fuerzas tangenciales, dirección y sentido, de la cáscara cilíndrica larga. Tonda Magallón J.A. (1974) Cascarones de Concreto. Capítulo V, figura 86, p. 46.

$$
D=\frac{R^{2} t \varphi}{t 2 R \operatorname{sen} \frac{\varphi}{2}}=R \frac{\varphi}{2 \operatorname{sen} \frac{\varphi}{2}}
$$

o bien:

$$
D=R \frac{S_{\varphi}}{C_{\varphi}}
$$

que resulta ser la expresión inversa a la del centro de gravedad:

$$
\overline{O G}=R \frac{C_{\varphi}}{S_{\varphi}}
$$

De la expresión [31], se concluye que:

$$
\frac{S_{\varphi}}{C_{\varphi}}>1
$$

por lo que la resultante de las fuerzas tangenciales, Rt, se ubicará fuera de la sección de la cáscara (Figura 8), al ser D > R.

El momento transversal, cuya expresión se trata de hallar, producido por la resultante será por tanto (Figura 8):

$$
M_{t \varphi}=R_{t} d
$$

Como gracias a las expresiones anteriores la distancia, D, quedó determinada, entonces:

$$
d=D-R \cos \frac{\varphi}{2}=R \frac{\varphi}{2 \operatorname{sen} \frac{\varphi}{2}}-R \cos \frac{\varphi}{2}
$$

Sustituyendo [27] y [35] en [34], se obtiene:

$$
M_{t \varphi}=\left[t 2 R \operatorname{sen} \frac{\varphi}{2}\right]\left[R \frac{\varphi}{2 \operatorname{sen} \frac{\varphi}{2}}-R \cos \frac{\varphi}{2}\right]
$$

Simplificando, se consigue finalmente la siguiente expresión:

$$
M_{t \varphi}=2 R^{2} \operatorname{tsen} \frac{\varphi}{2}\left(\frac{\varphi}{2 \operatorname{sen} \frac{\varphi}{2}}-\cos \frac{\varphi}{2}\right)=R^{2} t\left(\varphi-2 \operatorname{sen} \frac{\varphi}{2} \cos \frac{\varphi}{2}\right)
$$

$$
M_{t \varphi}=R^{2} t(\varphi-\operatorname{sen} \varphi)
$$

2.3. Momentos transversales debidos a la fuerza horizontal, Ho, o/y fuerza vertical, Vo, en el arranque de la cáscara.

En el caso de una única cáscaras continua, o en la última cáscara de una serie paralela de ellas, pueden aparecen fuerzas horizontales, $\mathrm{Ho}$, o/y verticales, Vo, en los arranques de la cáscara (Figura 9).

Como indica Tonda, si se trata de una fuerza horizontal ocasionada por la unión horizontal entre dos cáscaras, entonces se tratará de una fuerza de tracción por lo que provocará un momento positivo; cuya expresión vendrá dada por:

$$
M_{H_{\mathrm{o}}}=H_{\mathrm{o}} h_{\varphi}
$$

donde (Figura 9):

$$
\cos \alpha=\frac{x}{R} ; \quad \cos (\alpha-\varphi)=\frac{y}{R}
$$

por lo que:

$$
h_{\varphi}=y-x=R \cos (\alpha-\varphi)-R \cos \alpha=R[\cos (\alpha-\varphi)-\cos \alpha][41]
$$

que sustituyendo en la expresión [39] se obtiene:

$$
M_{h_{\mathrm{o}}}=H_{\mathrm{o}} R[\cos (\alpha-\varphi)-\cos \alpha]
$$

De igual manera, Tonda obtiene la expresión para el momento transversal producido por la presencia de una carga vertical, Vo, en el arranque de la cáscara. La expresión del citado momento transversal será (Figura 9):

$$
M_{V_{o}}=V_{\mathrm{o}} d_{\varphi}
$$

donde:

$$
d_{\varphi}=R \operatorname{sen} \alpha-R \operatorname{sen}(\alpha-\varphi)=R[\operatorname{sen} \alpha-\operatorname{sen}(\alpha-\varphi)][44]
$$

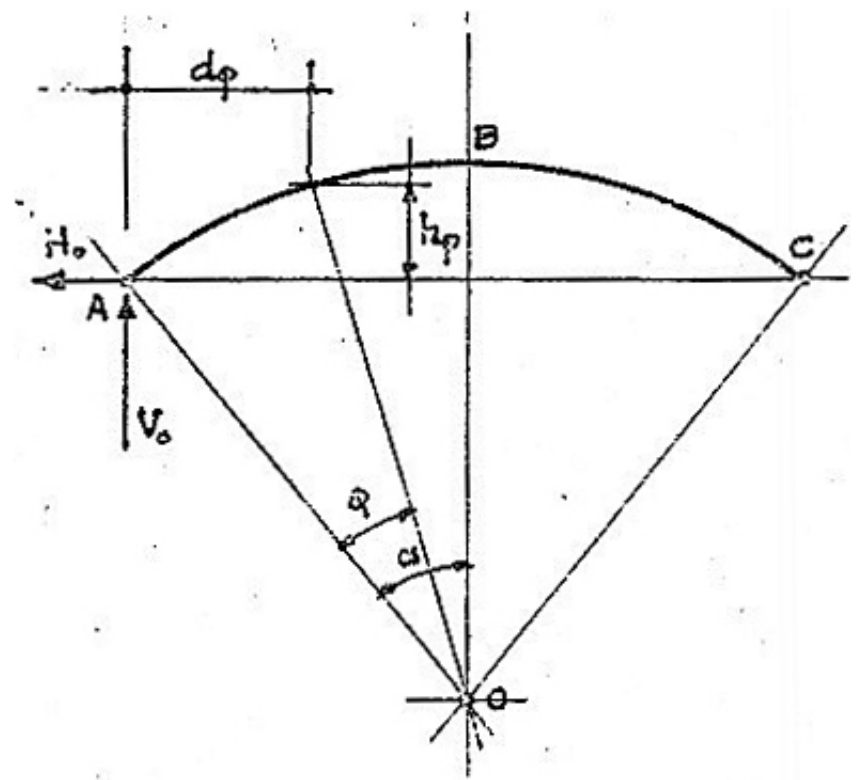

Figura 9. Representación de la fuerza horizontal y vertical en el arranque de la cáscara cilíndrica larga. Tonda Magallón J.A. (1974). Cascarones de Concreto. Capítulo V, figura 87, p. 49. 
que sustituyendo en la expresión [43] se obtiene:

$$
M_{V_{\mathrm{o}}}=V_{\mathrm{o}} R[\operatorname{sen} \alpha-\operatorname{sen}(\alpha-\varphi)]
$$

La existencia de continuidad transversal en las cáscaras cilíndricas largas puede evitar deformaciones excesivas, gracias a la acción de la reacción horizontal y del momento de empotramiento, transmitidos recíprocamente de una cáscara a otra; disminuyendo en forma apreciable los valores finales de los momentos transversales. Dichas deformaciones, por el contrario, sí podrían producirse en el caso de cáscaras aisladas.

Por último, para obtener los momentos transversales finales, habría que calcular las expresiones anteriormente halladas [26], [38], [42] y [45] en los diferentes puntos de la sección transversal de la cáscara, donde se desee calcular el valor final.

El valor de la fuerza horizontal, Ho, y de la fuerza vertical, Vo, en el arranque de la cáscara se deben calcular de tal modo que anulen el momento transversal producido tanto por el peso propio de la cáscara, como por las fuerzas tangenciales en el punto de coronación de la misma (punto B en la figura 9). Es decir, de nuevo, gracias al empleo del equilibrio plástico en la estructura, se puede obtener el resultado deseado.

En un ejemplo práctico resuelto por J. A. Tonda (7), éste calcula una cáscara cilíndrica larga de $30 \mathrm{~m}$ de longitud, libremente apoyada, con una anchura transversal de $8 \mathrm{~m}$, altura de coronación de $4 \mathrm{~m}$ (directriz semicircular) y continua a otras 5 cáscaras más, de idénticas propiedades.

Tras todo el desarrollo matemático, y siguiendo el procedimiento explicado anteriormente, Tonda dibuja el diagrama de los momentos transversales finales, obtenidos a intervalos de $5^{\circ}$ desde el arranque de la cáscara hasta la clave de ésta (Figura 10).

Como en todas las gráficas de momentos transversales finales, obtenidas en cáscaras cilíndricas por medio del equilibrio plástico de fuerzas, la sección transversal de la cáscara trabaja como si de un arco con tres articulaciones se tratara.
Una vez obtenido el valor del momento transversal final más elevado, que en la figura 10 correspondería al valor de $58,71 \mathrm{Kgm}$, se pasaría a calcular el refuerzo necesario para contrarrestarlo.

Lo importante del método, tal y como se ha descrito, reside en que se trata de un análisis de equilibrio de fuerzas, sumamente sencillo y que nada tiene que ver ni con los desarrollos matemáticos, ni con la complejidad de estos marcados por la Teoría de la Elasticidad.

\section{Esfuerzos cortantes:}

El esfuerzo cortante unitario del hormigón armado viene determinado por la expresión:

$$
v_{c}=\frac{t}{e}=\frac{V_{n}}{2 Z e}
$$

donde $e$ representa el espesor de la cáscara y, al igual que ocurre en una viga, el cortante se determina por:

$$
V_{n}=\frac{\omega_{b} L}{2}
$$

donde L representa la longitud de la cáscara.

Por otro lado, el esfuerzo cortante a lo largo del arco viene determinado por:

$$
Z_{m}=\frac{3}{4} \frac{V_{n}}{f}
$$

donde $f$ es la altura del arco de la sección transversal de la cáscara.

Tonda establece que si sucede:

$$
V_{c}=\frac{Z_{m}}{e}<4 \mathrm{~kg} / \mathrm{cm}^{2}
$$

entonces habrá que reforzar el hormigón con más área de refuerzo, obtenida por medio de la siguiente expresión:

$$
F_{e}=\frac{t}{\sigma_{e}}
$$

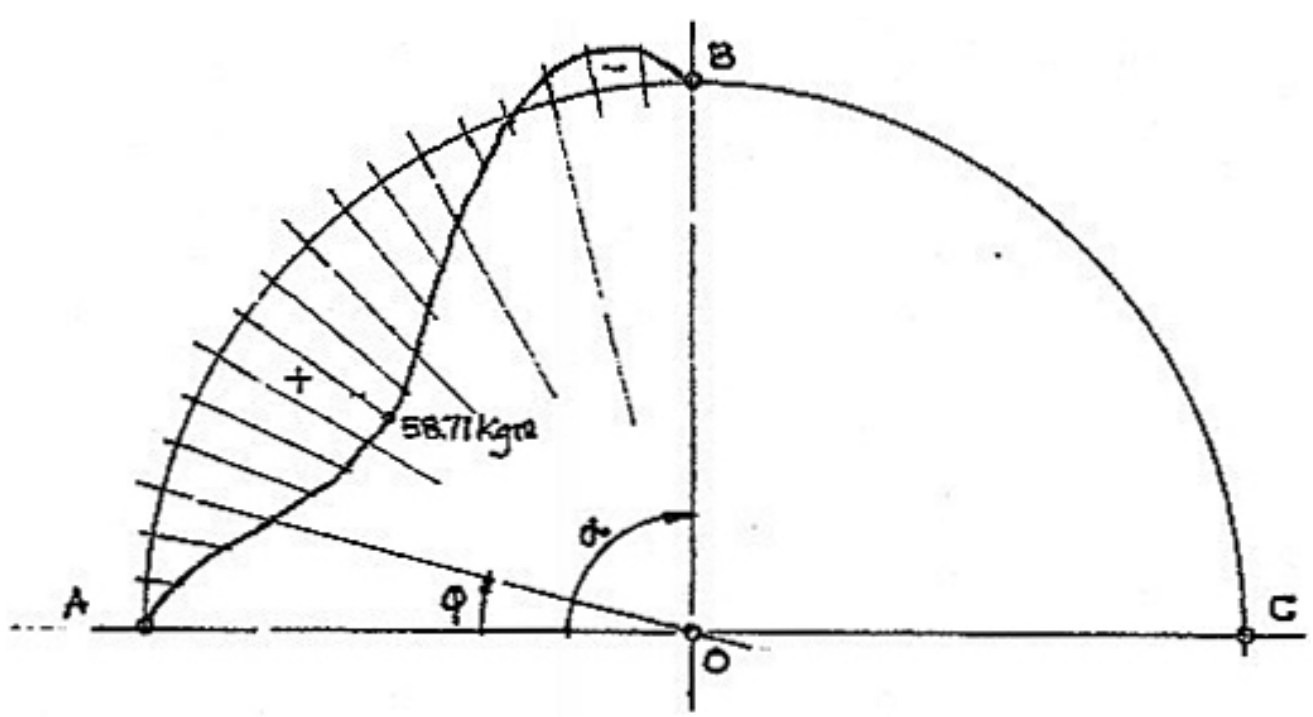

Figura 10. Diagrama de momentos transversales finales en la cáscara cilíndrica larga Tonda Magallón J.A. (1974). Cascarones de Concreto. Capítulo V, figura 95, p. 53. 


\section{CONCLUSIONES}

Para poder obtener todas las magnitudes hiperestáticas, producidas en el proceso de cálculo de una cáscara cilíndrica larga, utilizando la Teoría de la Elasticidad es necesario realizar un desarrollo matemático altamente complejo, a la vez que incierto. Sin embargo, utilizando el método de la viga, aplicado al cálculo de estas tipologías estructurales por J.A. Tonda, el problema se reduce a obtener un estado de equilibrio en la cáscara, con un enfoque plástico de éste, considerando la característica de cedencia del hormigón armado y, por tanto, olvidándose de introducir consideraciones de compatibilidad o deformación en el cálculo.

El método de la viga proporciona una solución de equilibrio que, sí la cáscara está constituida con un material dúctil y en ausencia de problemas de inestabilidad, resulta ser una solución segura; siempre y cuando se satisfaga la condición de cedencia del hormigón armado.

Por medio de transferencia de esfuerzos, de las zonas más solicitadas a las que lo están menos, se consigue el estado de equilibrio en la cáscara cilíndrica larga. Todo ello dependiendo de la geometría transversal de la cáscara, la ubicación de la fibra neutra y las diferentes disposiciones que se elija para la armadura. Por tanto el estado de equilibrio, así obtenido, es una solución al problema, pero no la única. Cualquier estado de la estructura en que se produzca el equilibrio de las fuerzas puede ser objeto de estudio, por lo que el calculista podía centrarse en estudiar la seguridad de la cáscara en cada uno de ellos.

\section{REFERENCIAS}

(1) Finsterwalder, U. (1932). Die Theorie der kreiszylindrischen Schalengewölbe System Zeiss-Dywidag und ihre anwendung auf die Grossmarkthalle in Budapest. Journal of Bridge and Structural Engineering. Primer Congreso IABSE, París: 127-152.

(2) Dischinger, Fr. (1935). Die strenge Theorie der Kreiszylinderschale in ihrer Anwendung auf die Zeiss-Dywidag-Schalen. Beton u. Eisen, (34): 257-264, 283-294.

(3) Jakobsen, A.Aas. (1941). Einzellasten auf Kreiszylinderschalen. Der Bauingenieur (22): 343-346.

(4) Johansen, K. W. (1944). Skalkonstruktion paa Radiohuset. Bygningsstatiske Meddelelser, (15): 1-26.

(5) Lundgren, H. (1949). Cylindrical Shells. Volumen I: Cylindrical Roofs. The Danish Techical Press the Institution of Danish Civil Engineers.

(6) Kazinczy, G.v. (1949). Beräkning av cylindriska skal med hänsyn till den armerade betongens egenskaper. Betong, (34): 239-261.

(7) Tonda, J. A. (1973). Cascarones de Concreto. Instituto Mexicano del Cemento y del Concreto A.C. 